PUPT-1286

September, 1991

\title{
QUANTUM SOLITONS IN AFFINE TODA FIELD THEORIES
}

\author{
Timothy Hollowood* \\ Joseph Henry Laboratories, Department of Physics, \\ Princeton University, Princeton, N.J. 08544
}

\begin{abstract}
The spectra of $A_{r}$ affine Toda field theories with imaginary coupling constant, are investigated. Soliton solutions are found, which, despite the non-unitary form of the Lagrangian, have real classical masses and are stable to small perturbations. The quantum corrections to the soliton masses are determined, to lowest order in $\hbar$. The solitons have the same spectrum as the fundamental Toda particles; a feature that is preserved in the quantum theory.
\end{abstract}

\footnotetext{
* Address after Oct. 1, 1991: Dept. of Theoretical Physics, Oxford, U.K.
} 


\section{Introduction}

Amongst the conformal field theories in two dimensions, there exists a much simpler class of theories, the so-called 'minimal theories', whose spectra consist of a finite number of representations of the - possibly extended - conformal algebra [1]. For example, there are the theories where the conformal algebra is extended to the $W_{r+1}$-algebras [2]. For each $r$ there exists a whole set of such theories whose central charges are conveniently parameterized in terms of a constant $\alpha$ :

$$
c=r\left\{1+(r+1)(r+2)\left(\alpha+\frac{1}{\alpha}\right)^{2}\right\} .
$$

For $c \geq r-$ so $\alpha$ is real - the theories are unitary, but not minimal. For $c<r-$ so $\alpha$ is purely imaginary - the situation is more complicated: when $\alpha^{2}$ is rational the theory is minimal, since a finite set of representations decouple from the complete Hilbert space. Furthermore, when $\alpha^{2}$ takes the particular rational values

$$
\alpha^{2}=-\frac{r+1+k}{r+2+k}
$$

where $k$ is a positive integer, the theory is, in addition, unitary. So, although a generic theory for which $c<r$ is non-unitary, there exists a set of measure zero unitary theories, when the coupling constant takes the rational values of (2).

This paper constitutes a study of the analogue of this phenomenon in the space of massive two-dimensional theories. In fact, we shall consider the massive theories which are thought to describe the $W_{r+1}$-conformal field theories perturbed by the particular relevant operator which preserves the integrability of the theory [3]. With $\alpha$ as in (2), the operator has dimension $(k+1) /(r+k+1)$. A deformation which preserves integrability is very special, and suggests that the resulting massive theory may be tractable.

There is evidence to suggest that these integrable theories are actually affine Toda field theories [4]. The case when $r=1$, which is the sine-Gordon theory, has been studied in some detail [5], however, the more theories for more general $r$ have not been the subject of much study - one of the reasons being that they are manifestly non-unitary. We show that although the theories are non-unitarity, there does exist a sector of soliton excitations which 
appears to be unitary. This suggests that the soliton spectrum is the massive remanent of the primary conformal fields of the minimal conformal field theory which describes the ultra-violet scaling limit of the theory.

The Lagrangian density of an affine Toda field theory - for a simply-laced Lie algebra - can be written in a number of ways: for instance

$$
\mathcal{L}=\frac{1}{2}\left(\left(\partial_{t} \phi\right)^{2}-\left(\partial_{x} \phi\right)^{2}\right)-\frac{m^{2}}{\gamma^{2}} \sum_{j=0}^{r} n_{j}\left(e^{\gamma \alpha_{j} \cdot \phi}-1\right)
$$

The field $\phi(x, t)$ is an $r=\operatorname{rank}(g)$ vector, where $g$ is a simply-laced Lie algebra, and consequently an algebra of type $A, D$ or $E$. The inner products are taken with respect to the Killing form of $g$ restricted to the Cartan subalgebra. The $\alpha_{j}$ 's, for $j=1, \ldots, r$ are the simple roots of $g ; \alpha_{0}$ is the extended root (minus the highest root of $g$ ). The fact that the extended root is included in the sum, distinguishes the affine theories from the non-affine ones. The $\alpha_{j}$ 's are linearly dependent:

$$
\sum_{j=0}^{r} n_{j} \alpha_{j}=0, \quad n_{0}=1
$$

which serves to define the integers $n_{j} ; \gamma$ and $m$ are constants. The quantum field theory defined by (3), is then thought to describe the integrable perturbation of the $W(g)$-algebra conformal field theory ${ }^{1}$, with the identification $\gamma=\sqrt{4 \pi} \alpha$. Notice that the particular value of $\gamma$ required to reproduce the minimal theories is actually imaginary. This implies that the Lagrangian density is no longer real - except for $g=A_{1}$, when it is the sine-Gordon theory. Prima facie, this would imply that the quantum field theory defined by (3) would be non-unitary. To mirror the situation at the critical point, we propose that when $\gamma^{2} / 4 \pi$ takes the rational values of (2), the Toda theory admits a unitary restriction. With this as the ultimate goal we now pursue the more modest aim of understanding the spectrum of the Toda theory with imaginary coupling constant $\gamma$.

Before proceeding it is worth briefly reviewing the properties of the quantum theory for real values of $\gamma$. The approach that has been adopted in the literature entails considering (3) in the weak coupling limit, and then comparing conjectured forms for the full $S$ matrix with the weak coupling perturbation expansion [6]. The reason why this strategy is feasible is because the theory is expected to be integrable also at the quantum level, and

\footnotetext{
1 The $W_{r+1}$-algebra theories are those for which $g=A_{r}$.
} 
so the $S$-matrix should be factorizable and also, in this instance, purely elastic [6]. The weak coupling expansion involves expanding (3) in powers of $\gamma$ about the minimum of the potential at $\phi=0$; the first two terms are

$$
V(\phi)=\frac{m^{2}}{2} \sum_{j=0}^{r} n_{j}\left(\alpha_{j} \cdot \phi\right)^{2}+\frac{m^{2} \gamma}{6} \sum_{j=0}^{r} n_{j}\left(\alpha_{j} \cdot \phi\right)^{3}+O\left(\gamma^{2}\right)
$$

In this limit, the theory describes a set of $r$ particles - the fundamental Toda particles whose masses are given by $2 m \sqrt{\zeta}$, where the $\zeta$ 's are the the eigenvalues of the matrix

$$
\sum_{j=0}^{r} n_{j} \alpha_{j} \otimes \alpha_{j}
$$

interacting via a set of three-point couplings defined by the term of order $\gamma$. The higher order terms describe corrections at various powers of $\gamma$. The first remarkable fact is that in the one-loop approximation all the masses are renormalized by an overall constant [6]. (This result is peculiar to the simply-laced theories.) On the basis of this, the first conjecture is that the mass ratios are preserved to all orders in $\gamma$. Furthermore, there are no additional states, so the quantum theory preserves the classical spectrum. For example, in the $A_{r}$ theories the classical masses are

$$
m_{a}=2 m \sin \left(\frac{\pi a}{r+1}\right), \quad a=1,2, \ldots, r
$$

and in the quantum theory the bare mass $m$ is replaced by a renormalized mass $m_{0}$. Recently, the conjectured $S$-matrix of the fundamental particles has been written in an elegant universal way using properties of the root system of the Lie algebra [7].

If $\gamma$ is imaginary, say $\gamma=i \beta$ for real $\beta$, then a naïve analytic continuation of the particle $S$-matrix would lead one to conclude that the spectrum discussed above cannot be complete because of the appearance of new poles on the physical strip, indicating the existence of new particles states. Moreover, some the the residues of the $S$-matrix, at the poles on the physical-strip, change sign - the hallmark of non-unitarity. In fact, we shall argue that the spectrum of the theory is actually much richer than this, because with imaginary coupling constant the theory also admits solitons. The solitons are present at the classical level, and correspond to static solutions of the equations of motion, in some frame of reference, with finite energy. It is straightforward to see why solitons will exist: with imaginary coupling constant the potential of the theory is periodic $\phi \sim \phi+(2 \pi / \beta) \omega$, 
where $\omega$ is an element of the weight lattice $\Lambda^{\star}$ of $g$. So the field $\phi$ takes values on the maximal torus of $g$. Solitons correspond to field configurations which wind around the non-trivial cycles of the maximal torus, as $x$ goes from $-\infty$ to $\infty$. Thus the solitons are 'kink' solutions, generalizing the situation for the sine-Gordon theory. What is not clear - since the potential is complex - is that the classical solitons have a real positive energy, and that they are stable to small perturbations. Remarkably both the former and latter are true for the $A_{r}$ theories, as we shall show. It turns out that the mass spectrum of solitons is equal to (7), up to an overall factor, although the solitons carry an additional quantum number which measures the topological charge. We further show that quantum corrections do not destroy this structure.

The paper is organized as follows. In $\S 1$, the classical theory is considered, multiple soliton solutions are constructed and the classical soliton masses are calculated. In $\S 2$ the quantum corrections to the soliton masses are calculated. $\S 3$ contains some comments.

\section{The Classical Solitons}

In this section we will construct classical soliton solutions of the $A_{r}$ theories described by (3) and calculate their masses.

\subsection{Multiple Soliton Solutions}

Our approach to constructing the soliton solutions follows the methods developed by R. Hirota for finding explicit expressions for the multi-soliton solutions of integrable equations [8]. There are alternative formalisms for finding these solutions, most notably the inverse scattering method, however, for this particular task the Hirota method is far superior; its elegance being manifested in a subtle kind of non-linear superposition principle for soliton solutions, which easily allows one to construct multiple soliton solutions. The method is intimately connected with the tau-function approach to integrable theories, of which an up to date survey may be found in [9].

Before more general theories are treated, it is useful to recall some properties of the sine-Gordon theory, which is obtained by setting $r=1$. With the definition $\psi=\sqrt{2} \phi$, the 
equation of motion, which follows from (3), is

$$
\left(\partial_{t}^{2}-\partial_{x}^{2}\right) \psi+\frac{4 m^{2}}{\beta} \sin \beta \psi=0
$$

It has been known for a long time (see [10] for example) that this equation admits a soliton solution of the form

$$
\psi=\frac{4}{\beta} \tan ^{-1}\left(e^{\sigma(x-v t-\xi)}\right)
$$

where $\sigma, v$ and $\xi$ are constants, and $\sigma^{2}\left(1-v^{2}\right)=4 m^{2}$. The anti-soliton corresponds to the negative of the above. (The direct method was applied to the theory by R. Hirota in [11].)

We now turn to the more general theories. The crucial step of the Hirota method is a change of variables which converts the equation of motion to an equation of 'Hirota bilinear type' (to use the parlance of the subject). The appropriate change of variables is not obvious; fortunately we are guided by the change of variables which is used for the Toda lattice equations, which are identical to the Toda field theory equations except that in the former the field only depends on $t[12]$. We are led to consider

$$
\phi=-\frac{1}{i \beta} \sum_{j=0}^{r} \alpha_{j} \log \tau_{j}
$$

where, for the moment, we are considering a general simply-laced theory. The new variables are the tau-functions for this theory. In terms of the $\tau_{j}$ 's, the equations of motion become

$$
\sum_{j=0}^{r} \alpha_{j}\left(\frac{\ddot{\tau}_{j} \tau_{j}-\dot{\tau}_{j}^{2}-\tau_{j}^{\prime \prime} \tau_{j}+\tau_{j}^{\prime 2}}{\tau_{j}^{2}}\right)=m^{2} \sum_{j=0}^{r} n_{j} \alpha_{j} \prod_{k=0}^{r} \tau_{k}^{-\alpha_{j} \cdot \alpha_{k}}
$$

(Here, dot denotes a $t$-derivative and prime an $x$-derivative.) This equation can be decoupled to give the collection of equations

$$
\ddot{\tau}_{j} \tau_{j}-\dot{\tau}_{j}^{2}-\tau_{j}^{\prime \prime} \tau_{j}+\tau_{j}^{\prime 2}=m^{2} n_{j}\left(\prod_{k=0}^{r} \tau_{k}^{A_{j k}}-\tau_{j}^{2}\right)
$$

The matrix with elements $A_{j k}$ is the adjacency matrix ${ }^{2}$ for the Dynkin diagram of the affine algebra $g^{(1)}$. In decoupling (1.3), the coefficient of $\tau_{j}^{2}$ has been fixed without loss of generality.

2 This is the matrix which encodes the connectivity of the Dynkin diagram, such that $A_{j j}=0$, and $A_{j k}=1$ if the $j^{t h}$ and $k^{t h}$ spot are joined, i.e. $\alpha_{j} \cdot \alpha_{k}=-1$. 
When $g=A_{r}$ the equations are

$$
\ddot{\tau}_{j} \tau_{j}-\dot{\tau}_{j}^{2}-\tau_{j}^{\prime \prime} \tau_{j}+\tau_{j}^{\prime 2}=m^{2}\left(\tau_{j-1} \tau_{j+1}-\tau_{j}^{2}\right) .
$$

The label on $\tau_{j}$ is to be understood modulo $r+1$, owing to the periodicity of the extended $A_{r}$ Dynkin diagram. Those familiar with the Hirota method will identify (1.5) as being of a 'bilinear type', whereas, on the contrary, for the other simply-laced algebras this does not seem to be the case. We do not consider these other algebras further, however, we will make some comments about the $D_{4}$ theory in $\S 3$.

To find multi-soliton solutions of the equations, the Hirota method starts by considering a series expansion in some arbitrary parameter $\epsilon$, which will be set to 1 at the end of the calculation. So

$$
\tau_{j}=1+\epsilon \tau_{j}^{(1)}+\epsilon^{2} \tau_{j}^{(2)}+\cdots .
$$

A solution describing $N$ solitons results from terminating (1.6) by setting $\tau_{j}^{(a)}=0$ for $a>N$. The fact that the series can be terminated is a crucial property of Hirota bilinear equations. Furthermore, one of the nice features of this approach is that the solutions have a very universal form for all integrable theories. The one-soliton solution has $\tau_{j}^{(a)}=0$ for $a>1$, and $\tau_{j}^{(1)}$ satisfies the linear differential equation

$$
\ddot{\tau}_{j}^{(1)}-\tau_{j}^{(1) \prime \prime}=m^{2}\left(\tau_{j+1}^{(1)}+\tau_{j-1}^{(1)}-2 \tau_{j}^{(1)}\right),
$$

and the condition

$$
\tau_{j+1}^{(1)} \tau_{j-1}^{(1)}=\tau_{j}^{(1) 2}
$$

which are solved by

$$
\tau_{j}^{(1)}=\exp \{\sigma(x-v t-\xi)\} \delta_{j},
$$

for constants $\sigma, v, \xi$ and $\delta_{j}$. The constants $\delta_{j}$ have to satisfy the recursion relations

$$
\delta_{j+1} \delta_{j}^{-1}=\delta_{j} \delta_{j-1}^{-1}
$$

and the periodicity requirement $\delta_{j+r+1}=\delta_{j}$. It is straightforward to solve for $\delta_{j}$, yielding

$$
\delta_{j}=\omega^{j}
$$

where $\omega$ is an $(r+1)^{\text {th }}$ root of unity. So there are $r$ non-trivial solutions for which $\omega=$ $\omega_{a} \equiv \exp 2 \pi i a /(r+1)$. In addition the constants $\sigma$ and $v$, and the integer $a \in\{1,2, \ldots, r\}$, have to satisfy

$$
\sigma^{2}\left(1-v^{2}\right)=4 m^{2} \sin ^{2}\left(\frac{\pi a}{n+1}\right)=m_{a}^{2}
$$


where the $m_{a}$ 's are the classical masses of the fundamental particles defined in (7). (1.12) is just an expression of the fact that the theory is Lorentz invariant; the faster a soliton goes the narrower it becomes.

The one-soliton solution is therefore

$$
\bar{\phi}_{(a)}(x, t)=-\frac{1}{i \beta} \sum_{j=0}^{r} \alpha_{j} \log \left(1+e^{\sigma(x-v t-\xi)} \omega_{a}^{j}\right) .
$$

The solution is in general complex, however, the asymptotic values of $\bar{\phi}_{(a)}$ as $x \rightarrow \pm \infty$ are real. If we assume that $\sigma>0$, as $x \rightarrow-\infty \phi \rightarrow 0$, and as $x \rightarrow \infty$

$$
\phi \rightarrow \frac{2 \pi}{\beta}\left(\lambda_{a} \bmod \Lambda\right)
$$

where $\lambda_{a}$ is the $a^{\text {th }}$ fundamental weight ${ }^{3}$ and $\Lambda$ is the root lattice of $g$. We define the topological charge a configuration to be

$$
t=\frac{\beta}{2 \pi} \int_{-\infty}^{\infty} d x \partial_{x} \phi=\frac{\beta}{2 \pi}\left(\lim _{x \rightarrow \infty}-\lim _{x \rightarrow-\infty}\right) \phi(x) .
$$

So the one-soliton solution $\bar{\phi}_{(a)}$ has a topological charge which is equal to the $a^{\text {th }}$ fundamental weight, up to a root. The actual value depends on a choice for the branch-cuts of the logarithms in (1.13). A careful analysis of the possibilities shows that the possible topological charges of the one-soliton solution $\bar{\phi}_{(a)}$ fill out the weights of the $a^{\text {th }}$ fundamental representation.

When $g=A_{1}$ the solution can be chosen to real by choosing $\exp \sigma \xi=-i \exp \sigma \xi^{\prime}$ with $\xi^{\prime}$ real, i.e.

$$
\tau_{1}=\tau_{0}^{\star}=1-i e^{\sigma\left(x-v t-\xi^{\prime}\right)}
$$

reproducing the sine-Gordon soliton solution (1.1).

Multi-soliton solutions result from terminating (1.6) at a higher power of $\epsilon$. It might be thought that the complexity of the solution increases rapidly with the number of solitons; however, this is not the case due to a subtle form of non-linear superposition principle which underlies the Hirota method. The two soliton solution illustrates the situation. The solution depends on a set of data $\left\{\sigma_{p}, v_{p}, \xi_{p}, a_{p}\right\}$, with $p=1,2 ; v_{p}$ are the velocities, $\xi_{p}$ are the position offsets and $\sigma_{p}$ are the size parameters of the solitons. It is useful to define

$$
\Phi_{j}^{(p)}=\sigma_{p}\left(x-v_{p} t-\xi_{p}\right)+\log \left(\omega_{a_{p}}^{j}\right)
$$

\footnotetext{
3 The fundamental weights are dual to the roots: $\alpha_{j} \cdot \lambda_{k}=\delta_{j k}$.
} 
then the form of the two soliton solution, after setting $\epsilon=1$, is

$$
\tau_{j}=1+e^{\Phi_{j}^{(1)}}+e^{\Phi_{j}^{(2)}}+A e^{\Phi_{j}^{(1)}+\Phi_{j}^{(2)}} .
$$

Substituting this ansatz into (1.5) fixes the 'interaction constant'

$$
A=-\frac{\left(\sigma_{1}-\sigma_{2}\right)^{2}-\left(\sigma_{1} v_{1}-\sigma_{2} v_{2}\right)^{2}-4 m^{2} \sin ^{2}\left(\frac{\pi}{n+1}\left(a_{1}-a_{2}\right)\right)}{\left(\sigma_{1}+\sigma_{2}\right)^{2}-\left(\sigma_{1} v_{1}+\sigma_{2} v_{2}\right)^{2}-4 m^{2} \sin ^{2}\left(\frac{\pi}{n+1}\left(a_{1}+a_{2}\right)\right)}
$$

The expression for the multi-soliton solution builds on this elegant form: for example, the three soliton solution is

$$
\begin{aligned}
\tau_{j}= & 1+e^{\Phi_{j}^{(1)}}+e^{\Phi_{j}^{(2)}}+e^{\Phi_{j}^{(3)}}+A^{(12)} e^{\Phi_{j}^{(1)}+\Phi_{j}^{(2)}}+ \\
& A^{(13)} e^{\Phi_{j}^{(1)}+\Phi_{j}^{(3)}}+A^{(23)} e^{\Phi_{j}^{(2)}+\Phi_{j}^{(3)}}+A^{(12)} A^{(13)} A^{(23)} e^{\Phi_{j}^{(1)}+\Phi_{j}^{(2)}+\Phi_{j}^{(3)} .}
\end{aligned}
$$

The general $N$ soliton solution can be written in the compact form

$$
\tau_{j}=\sum_{\left\{\mu_{p}\right\}=0,1} \exp \left(\sum_{p=1}^{N} \mu_{p} \Phi_{j}^{(p)}+\sum_{p, q=1}^{N} \mu_{p} \mu_{q} \gamma^{(p q)}\right),
$$

where $\gamma^{(p q)}=\log A^{(p q)}$. The sum is over the $2^{N}$ possibilities for which $\mu_{p}=0$ or 1 , for each $p$.

The multi-soliton solution have a natural physical interpretation. They represent the histories of a set of solitons which scatter off each other. To make this more precise consider the two soliton solution. Suppose that $\xi_{1}<\xi_{2}, v_{1}>v_{2}$, and $\sigma_{1}$ and $\sigma_{2}$ are both positive. Focus on the solution in the vicinity of the first soliton, i.e. $x \sim v_{1} t+\xi_{1}$. Initially, that is in the limit $t \rightarrow-\infty$, the solution is approximately

$$
\tau_{j} \simeq 1+e^{\Phi_{j}^{(1)}}
$$

and finally, i.e. as $t \rightarrow \infty$, the solution is approximately

$$
\tau_{j} \simeq e^{\Phi_{j}^{(2)}}\left(1+A e^{\Phi_{j}^{(1)}}\right) .
$$

In both the limits, the solution represents an isolated soliton, the only difference is that the final 'position offset' has been displaced: $\xi_{1} \longmapsto \xi_{1}+\ln A$. The fact that the velocities and form are unchanged by the collision is a characteristic feature of soliton scattering in an integrable field theory, reflecting the existence of the infinite set of integrals of motion. So asymptotically the solution represents two isolated solitons. What is not so apparent is that the topological charges of the solitons can be altered by the scattering (although the representations, i.e. $a_{1}$ and $a_{2}$, are fixed). To see this requires a rather detailed analysis, that will not be pursued here, of the positions of the branch-cuts of the logarithms. 


\subsection{Classical Soliton Masses}

The purpose of this section is to calculate the classical masses of the solitons that were constructed in the last section. The result is highly significant because it transpires that the ratios of the soliton masses are equal to the ratios of the masses of the fundamental particles (7). The result is that the one-soliton solution of (1.13), with a topological charge $t=\lambda_{a} \bmod \Lambda$, has classical mass

$$
M_{a}^{\mathrm{cl}}=\frac{4 m(r+1)}{\beta^{2}} \sin \left(\frac{\pi a}{r+1}\right)=\frac{2(r+1)}{\beta^{2}} m_{a} .
$$

To find the masses of the soliton solutions, either the energy

$$
M \gamma(v)=\int_{-\infty}^{\infty} d x\left(\frac{1}{2} \dot{\phi}^{2}+\frac{1}{2} \phi^{\prime 2}-\frac{m^{2}}{\beta^{2}} \sum_{j=0}^{r} n_{j}\left(e^{i \beta \alpha_{j} \cdot \phi}-1\right)\right)
$$

or the momentum

$$
M \gamma(v) v=-\int_{-\infty}^{\infty} d x \dot{\phi} \cdot \phi^{\prime}
$$

of a soliton must be calculated. (As usual, in the above $\gamma(v)=\left(1-v^{2}\right)^{-1 / 2}$.) The momentum is somewhat easier to calculate. In doing so it is useful to introduce the weights of the $(r+1)$-dimensional representation ${ }^{4}$ of $A_{r}$, and to define $\phi_{j}=e_{j} \cdot \phi$. The momentum is then

$$
P=-\int_{-\infty}^{\infty} d x \sum_{j=1}^{r+1} \dot{\phi}_{j} \phi_{j}^{\prime}=-\sum_{j=1}^{n+1} \int_{\phi_{j-}}^{\phi_{j+}} d \phi_{j} \dot{\phi}_{j}
$$

where $\phi_{j \pm}$ are the asymptotic values of $\phi_{j}(x, t)$ as $x \rightarrow \pm \infty$. Using the explicit expression for the one-soliton solution $\phi_{(a)}(x, t)$ in $(1.13)$ one finds

$$
\begin{aligned}
\dot{\phi}_{j} & =-\frac{1}{i \beta} \frac{\partial}{\partial t} \ln \left(\frac{\tau_{j}}{\tau_{j-1}}\right) \\
& =\frac{\sigma v}{i \beta}\left(\frac{\delta^{j} e^{\Phi}}{1+\delta^{j} e^{\Phi}}-\frac{\delta^{j-1} e^{\Phi}}{1+\delta^{j-1} e^{\Phi}}\right)
\end{aligned}
$$

4 The weights of the $(r+1)$-dimensional representation are $e_{j}$, for $j=1$ to $r+1$, with $\sum_{j=1}^{r+1} e_{j}=0$; in terms of which the roots are $\alpha_{j}=e_{j}-e_{j+1}$, with $e_{0} \equiv e_{r+1}$. 
where $\delta=\omega_{a}$ and $\Phi=\sigma(x-v t-\xi)$. This expression can be rewritten in terms of $\phi_{j}$ using the expressions

$$
\frac{\delta^{j} e^{\Phi}}{1+\delta^{j} e^{\Phi}}=-\frac{1}{1-\delta^{-1}}\left(\frac{1+\delta^{j-1} e^{\Phi}}{1+\delta^{j} e^{\Phi}}-1\right)=\frac{1}{1-\delta^{-1}}\left(1-e^{i \beta \phi_{j}}\right)
$$

and

$$
\frac{\delta^{j-1} e^{\Phi}}{1+\delta^{j-1} e^{\Phi}}=-\frac{1}{1-\delta}\left(\frac{1+\delta^{j} e^{\Phi}}{1+\delta^{j-1} e^{\Phi}}-1\right)=\frac{1}{1-\delta}\left(1-e^{-i \beta \phi_{j}}\right) .
$$

Using these (1.26) becomes

$$
\dot{\phi}_{j}=\frac{\sigma v}{i \beta}\left(\frac{1}{1-\delta} e^{-i \beta \phi_{j}}-\frac{1}{1-\delta^{-1}} e^{i \beta \phi_{j}}+\frac{1}{1-\delta^{-1}}-\frac{1}{1-\delta}\right)
$$

The utility of this expression is that $\dot{\phi}_{j}$ has been written solely in terms of $\phi_{j}$, and hence the integrals in (1.25) can now be performed. The constant terms in (1.29) do not contribute because $\sum_{j=1}^{r+1} \phi_{j}=0$. Therefore

$$
P=-\left.\sigma v \beta^{2} \sum_{j=1}^{r+1}\left(\frac{1}{1-\delta} e^{-i \beta \phi_{j}}+\frac{1}{1-\delta^{-1}} e^{i \beta \phi_{j}}\right)\right|_{\phi_{j-}} ^{\phi_{j+}}
$$

The evaluation of this expression is straightforward using the asymptotic expressions for $\phi_{j}$

$$
e^{i \beta \phi_{j}}= \begin{cases}\delta^{-1} & x \rightarrow \infty \\ 1 & x \rightarrow-\infty\end{cases}
$$

The following masses are found

$$
M_{a}^{\mathrm{cl}}=\frac{P}{\gamma(v) v}=\frac{2 \sigma(r+1)}{\gamma(v) \beta^{2}}=\frac{4 m(r+1)}{\beta^{2}} \sin \left(\frac{\pi a}{n+1}\right),
$$

where the relation (1.12) was employed to eliminate $\sigma$.

\section{Quantum Corrections to the Soliton Masses}

In this section we calculate the first quantum corrections to the soliton masses. We employ the semi-classical WKB method described in [13] for the kinks, or solitons, of the $\phi^{4}$ and sine-Gordon theories - see also the review in [10]. 
The dimensionless expansion parameter in our theory is $\hbar \beta^{2} / m^{2}$, and so the expansion in $\hbar$ coincides with the weak coupling expansion. For the sine-Gordon theory it is remarkable that the semi-classical WKB method, which gives the quantum corrections to the first order in $\hbar \beta^{2} / m^{2}$, is exact! The reason why the WKB method is exact for the sine-Gordon theory is, presumably, a consequence of its integrability. It seems plausible that the same is true for the affine Toda theories. In any case, we compute the mass corrections following exactly the same method as was used in [13] for the $\phi^{4}$ and sine-Gordon theories - the latter being included in our calculation as the special case for which $r=1$.

From now on we set $\hbar=1$. The WKB method of [13] is straightforward to apply in our situation since the one-soliton solution is, in its rest frame, time-independent. The idea is to compute the zero-point energy of the small oscillations around the classical solution. The sum over all the modes can be done when an infra-red regulator is introduced; this is most easily achieved by putting the theory in a box. The zero-point energy of the vacuum must then be subtracted. The resulting expression is independent of the size of the box, $L$, as $L \rightarrow \infty$; however, the final expression has an ultra-violet divergence. This divergence is not a problem of the soliton solution per se, rather, it is just a manifestation of the fact that the bare mass needs to be renormalized. This is achieved simply by normal-ordering the Lagrangian. The extra correction removes the divergence and the resulting finite residue which remains is then the mass correction.

To begin with, we must analyse the small perturbations around the one-soliton solution. If we write $\phi=\bar{\phi}_{(a)}+\eta$, where $\bar{\phi}_{(a)}$ is the static soliton solution in (1.13)- with $v=0$ and $\sigma=m_{a}$ - and expand in powers of $\eta$, to first order in $\eta$ we find

$$
\left(\partial_{t}^{2}-\partial_{x}^{2}\right) \eta+m^{2} \sum_{j=0}^{r} e^{i \beta \alpha_{j} \cdot \bar{\phi}_{(a)}} \alpha_{j}\left(\alpha_{j} \cdot \eta\right)=0
$$

For a generic field theory, it is impossible to find the solutions to the linearized equation of motion around a classical solution of the equations of motion. However, we are in the fortunate position of studying an integrable theory for which we can find the modes explicitly. Asymptotically, as $x \rightarrow \pm \infty,(2.1)$ becomes

$$
\left(\partial_{t}^{2}-\partial_{x}^{2}\right) \eta+m^{2} \sum_{j=0}^{r} \alpha_{j}\left(\alpha_{j} \cdot \eta\right)=0 .
$$

This is nothing but the linearized classical equation of motion of the theory around the 'vacuum' field configuration $\phi=0$. Since $\eta$ is an $r$-dimensional vector there are $r$ indepen- 
dent solutions of the full equation (2.1), with asymptotic form

$$
\eta_{b}(x, t) \underset{x \rightarrow \pm \infty}{\longrightarrow} e^{i\left(k x+\nu t+\delta_{ \pm}\right)} \sum_{j=0}^{r} \omega_{b}^{j} \alpha_{j}, \quad b=1, \ldots, r
$$

with frequency

$$
\nu^{2}=k^{2}+m_{b}^{2}
$$

which correspond to the fundamental particles modes of the Toda theory. We now proceed to find the exact solutions to the linearized equation (2.1). With (2.3) in mind, we are led to consider the two-soliton solution in eq. (1.18). The first soliton will be the static onesoliton solution that we are expanding around, the second will act as a small perturbation of the first. We take $\sigma_{2}=i k$ and $\sigma_{2} v_{2}=-i \nu$ and treat $\exp \sigma_{2} \xi_{2}$ as a small parameter, in which case

$$
\eta_{b}=-\frac{1}{i \beta} \sum_{j=0}^{r} \alpha_{j} \frac{\delta \tau_{j}}{\tau_{j}}
$$

where $\tau_{j}$ corresponds to the one-soliton solution and

$$
\delta \tau_{j}=e^{\Phi_{j}^{(2)}}+A e^{\Phi_{j}^{(1)}+\Phi_{j}^{(2)}}
$$

The explicit expression for the $b^{\text {th }}$ mode is therefore

$$
\eta_{b}(k ; x, t)=-\frac{1}{i \beta} \sum_{j=0}^{r} \alpha_{j}\left(\frac{1+A \omega_{a}^{j} e^{m_{a}(x-\xi)}}{1+\omega_{a}^{j} e^{m_{a}(x-\xi)}}\right) \omega_{b}^{j} e^{i(k x+\nu t)},
$$

with frequency $\nu^{2}=k^{2}+m_{b}^{2}$. The interaction parameter $A$ was found in (1.19), and for the present solution it is

$$
A(k)=\frac{m_{a}^{2}+m_{b}^{2}-m_{a-b}^{2}-2 i m_{a} k}{m_{a}^{2}+m_{b}^{2}-m_{a+b}^{2}+2 i m_{a} k} .
$$

It is straightforward to verify that the solutions (2.6) then have the correct asymptotic behaviour as predicted in (2.3).

In addition to the solutions found above, there is a zero-mode solution (having zero frequency) of the form

$$
\eta \propto\left(\frac{\partial \bar{\phi}_{(a)}}{\partial x}\right)
$$


This solution exists because the theory is translationally invariant and so there exists a whole family of one-soliton solutions which only differ in the position of the centre-ofmass. For the WKB method, it turns out that one can ignore this mode [13]; a more sophisticated time-dependent treatment shows that its effect is simply to ensure that the quantum soliton behaves as a relativistic particle, i.e. has energy levels $E=\sqrt{M^{2}+P^{2}}$, where $M$ is the quantum mass of the soliton and $P$ is its momentum.

Before we proceed to calculate the quantum mass of the soliton, it is worth pointing out that since there are no modes with imaginary frequency the solitons are classical stable. This is quite surprising given the fact that the potential of the theory is complex.

In order to calculate the zero-point energy of the modes it is necessary to introduce some finite boundary conditions, whence the modes become discrete and it is possible to enumerate them. The appropriate boundary conditions in this case are $\eta(x=0)=\eta(x=$ $L)=0$, where the centre-of-mass of the soliton lies between inside the box, and $L$ is much larger than the size of the soliton $\sim m_{a}^{-1}$. The solutions satisfying these boundary conditions are

$$
\eta_{b}\left(k_{m} ; x, t\right)-\eta_{b}\left(-k_{m} ; x, t\right)
$$

with

$$
k_{m} L+\rho_{b}\left(k_{m}\right)=\pi m, \quad m \in Z .
$$

where $\rho_{b}(k)=\operatorname{Im}(\log A(k))$. Notice that $\rho_{b}(-k)=-\rho_{b}(k)$.

The quantum correction to the soliton mass is then obtained by taking the zero-point energy of the modes around the soliton solution and subtracting the zero-point energy of modes around the vacuum, which satisfy (2.10) with $\rho=0$. That is

$$
\Delta M_{a}=\frac{1}{2} \sum_{b=1}^{r} \sum_{m \geq 0}\left(\sqrt{k_{m}^{2}+m_{b}^{2}}-\sqrt{\left(k_{m}+\rho_{b}\left(k_{m}\right) / L\right)^{2}+m_{b}^{2}}\right),
$$

where $k_{m}$ satisfies (2.10). Since $L$ will eventually be taken to infinity, we can expand in $L^{-1}$, the leading term in the sum being

$$
-\frac{k \rho_{b}(k)}{\sqrt{k^{2}+m_{b}^{2}}}=-\rho_{b}(k) \frac{d \epsilon_{b}(k)}{d k}
$$

with $\epsilon_{b}(k)=\sqrt{k^{2}+m_{b}^{2}}$. Now, we take $L \rightarrow \infty$ in which case we can replace the sum over $m$ by an integral over $k$ :

$$
\sum_{m \geq 0}=L\left\{\int_{-\infty}^{\infty} \frac{d k}{2 \pi}+O\left(L^{-1}\right)\right\}
$$


Therefore, the mass correction is

$$
\begin{aligned}
\Delta M_{a} & =-\sum_{b=1}^{r} \int_{-\infty}^{\infty} \frac{d k}{4 \pi} \rho_{b}(k) \frac{d \epsilon_{b}(k)}{d k} \\
& =-\frac{1}{4 \pi} \sum_{b=1}^{r}\left\{\left.\rho_{b}(k) \epsilon_{b}(k)\right|_{-\infty} ^{\infty}+\int_{-\infty}^{\infty} d k \epsilon_{b}(k) \frac{d \rho_{b}(k)}{d k}\right\} .
\end{aligned}
$$

Now as $k \rightarrow \pm \infty, \epsilon_{b}(k) \rightarrow|k|$ and

$$
\rho_{(b)}(k) \rightarrow \frac{1}{2 m_{a} k}\left(2 m_{a}^{2}+2 m_{b}^{2}-m_{a-b}^{2}-m_{a+b}^{2}\right),
$$

and thus the first contribution from (2.14) is

$$
\left.\sum_{b=1}^{r} \rho_{b}(k) \epsilon_{b}(k)\right|_{-\infty} ^{\infty}=\frac{1}{m_{a}} \sum_{b=1}^{r}\left(2 m_{a}^{2}+2 m_{b}^{2}-m_{a-b}^{2}-m_{a+b}^{2}\right) .
$$

The sum is straightforward to perform, the result being a contribution

$$
-\frac{m_{a}(r+1)}{2 \pi}
$$

to the mass. As it stands, the second contribution in (2.14) is not well defined because the integral has a logarithmic divergence. The problem is not due to any special nature of the soliton solution, indeed it is to be expected and is due to the fact that we have not renormalized the Lagrangian (3). We now pause to consider this aspect in more detail.

Mercifully, the renormalization process in a two-dimensional field theory is straightforward. The divergences can simply be removed by normal-ordering the Lagrangian. Working to lowest order in $\beta^{2}$ and introducing an ultra-violet cut-off $\Lambda$

$$
: e^{i \beta \alpha_{j} \cdot \phi}:=e^{i \beta \alpha_{j} \cdot \phi}\left(1+\frac{1}{2} \beta^{2} \sum_{a=1}^{r}\left(\zeta_{a} \cdot \alpha_{j}\right)^{2} \Delta_{a}+O\left(\beta^{4}\right)\right)
$$

where

$$
\Delta_{a}=\int_{-\Lambda}^{\Lambda} \frac{d k}{4 \pi} \cdot \frac{1}{\sqrt{k^{2}+m_{a}^{2}}}
$$

and $\zeta_{a}$ is the $a^{\text {th }}$ eigenvector of $\sum_{j=0}^{r} \alpha_{j} \otimes \alpha_{j}$ of eigenvalue $\left(m_{a} / m\right)^{2}$. Hence, to lowest order in $\beta^{2}$, the renormalized Lagrangian is equal to

$$
L=\frac{1}{2}\left(\left(\partial_{t} \phi\right)^{2}-\left(\partial_{x} \phi\right)^{2}\right)+\frac{1}{\beta^{2}} \sum_{j=0}^{r}\left(m^{2}+\partial m_{j}^{2}\right)\left(e^{i \beta \alpha_{j} \cdot \phi}-1\right) \text {, }
$$


with

$$
\partial m_{j}^{2}=\frac{1}{2}(m \beta)^{2} \sum_{a=1}^{r}\left(\zeta_{a} \cdot \alpha_{j}\right)^{2} \Delta_{a},
$$

and we have added a constant to bring the energy of the vacuum to zero. The presence of the additional terms clearly changes the energy of the soliton solution. These changes must be added to the quantum correction to the soliton mass. It might be thought that we should now consider the one-soliton solution for the renormalized Lagrangian; fortunately, this is unnecessary since the correction due to change in the form of the solution does not occur at the lowest order in $\beta^{2}$, precisely because the soliton solution is an extremum of the action.

Taking into account the counter-term from the renormalization, the final expression for the mass correction for the $a^{\text {th }}$ soliton is

$$
\begin{aligned}
\Delta M_{a}=-\frac{m_{a}(n+1)}{2 \pi}+\sum_{b=1}^{r} \int_{-\Lambda}^{\Lambda} \frac{d k}{4 \pi} \epsilon_{b}(k) \frac{d \rho_{b}(k)}{d k} \\
-\frac{1}{\beta^{2}} \sum_{j=0}^{r} \int_{-\infty}^{\infty} d x\left(e^{\left.i \beta \alpha_{j} \cdot \bar{\phi}_{(a)}-1\right) \partial m_{j}^{2} .}\right.
\end{aligned}
$$

We now pause to calculate the integral

$$
\int_{-\infty}^{\infty} d x\left(e^{i \beta \alpha_{j} \cdot \bar{\phi}_{(a)}}-1\right)
$$

Firstly

$$
e^{i \beta \alpha_{j} \cdot \bar{\phi}_{(a)}}=\prod_{k=0}^{r} \tau_{j}^{-\alpha_{j} \cdot \alpha_{k}}=\frac{\tau_{j-1} \tau_{j+1}}{\tau_{j}^{2}},
$$

where $\tau_{j}=1+\omega_{a}^{j} e^{m_{a}(x-\xi)}$ is the static one-soliton solution. Using (1.5) we see that

$$
\begin{aligned}
e^{i \beta \alpha_{j} \cdot \bar{\phi}_{(a)}-1} & =\frac{1}{m^{2}} \cdot \frac{-\tau_{j}^{\prime \prime} \tau_{j}+\tau_{j}^{\prime 2}}{\tau_{j}^{2}} \\
& =-\left(\frac{m_{a}}{m}\right)^{2} \cdot \frac{\omega_{a}^{j} e^{m_{a}(x-\xi)}}{\left(1+\omega_{a}^{j} e^{m_{a}(x-\xi)}\right)^{2}}
\end{aligned}
$$

The integral (2.23) may now be calculated explicitly yielding

$$
\left.\left(\frac{m_{a}}{m}\right)^{2} \cdot \frac{1}{m_{a}\left(1+\omega_{a}^{j} e^{m_{a}(x-\xi)}\right)}\right|_{-\infty} ^{\infty}=-\frac{m_{a}}{m^{2}}
$$


The important point about the resulting expression for the integral is that it is independent of $j$. The contribution to the mass correction (2.22) from the renormalization counter-term can now be simplified:

$$
\begin{aligned}
-\frac{1}{\beta^{2}} \sum_{j=0}^{r} \int_{-\infty}^{\infty} & d x\left(e^{i \beta \alpha_{j} \cdot \bar{\phi}_{(a)}}-1\right) \partial m_{j}^{2} \\
& =\frac{1}{2} m_{a} \sum_{b=1}^{r} \sum_{j=0}^{r}\left(\zeta_{b} \cdot \alpha_{j}\right)^{2} \Delta_{b} \\
& =\frac{1}{2} m_{a} \sum_{b=1}^{r}\left(\frac{m_{b}}{m}\right)^{2} \int_{-\Lambda}^{\Lambda} \frac{d k}{4 \pi} \cdot \frac{1}{\sqrt{k^{2}+m_{b}^{2}}}
\end{aligned}
$$

where we used the fact that $\zeta_{b}$ is an eigenvector of $\sum_{j=0}^{r} \alpha_{j} \otimes \alpha_{j}$ of eigenvalue $\left(m_{b} / m\right)^{2}$.

The expression for the mass correction is now

$$
\begin{aligned}
\Delta M_{a} & =-\frac{m_{a}(r+1)}{2 \pi}+m_{a} \sum_{b=1}^{r} \int_{-\Lambda}^{\Lambda} \frac{d k}{4 \pi}\left\{\frac{1}{2}\left(\frac{m_{b}}{m}\right)^{2} \cdot \frac{1}{\sqrt{k^{2}+m_{b}^{2}}}\right. \\
& \left.-\sqrt{k^{2}+m_{b}^{2}} \frac{4\left(m_{a}^{2}+m_{b}^{2}-m_{a+b}^{2}\right)}{\left(\left(m_{a}^{2}+m_{b}^{2}-m_{a+b}^{2}\right)^{2}+4 m_{a}^{2} k^{2}\right)}\right\} .
\end{aligned}
$$

Using the fact that

$$
\sum_{b=1}^{r}\left(2 m_{a}^{2}+2 m_{b}^{2}-m_{a-b}^{2}-m_{a+b}^{2}\right)=2 m_{a}^{2}(r+1),
$$

and

$$
\sum_{b=1}^{r}\left(\frac{m_{b}}{m}\right)^{2}=\operatorname{Tr}\left(\sum_{j=0}^{r} \alpha_{j} \otimes \alpha_{j}\right)=\sum_{j=0}^{r} \alpha_{j} \cdot \alpha_{j}=2(r+1),
$$

one can easily verify that for large $|k|$ the integrand behaves as $k^{-2}$ and so, as required, the logarithmic divergence is cancelled by the renormalization counter-term. We can now let the ultra-violet cut-off $\Lambda$ tend to infinity.

The remaining contribution is given in terms of a convergent integral:

$$
\Delta M_{a}=m_{a}\left\{-\frac{r+1}{2 \pi}+I_{a}\right\}
$$

where

$$
\begin{gathered}
I_{a}=\int_{-\infty}^{\infty} \frac{d k}{4 \pi} \sum_{b=1}^{r}\left\{-\sqrt{k^{2}+m_{b}^{2}} \cdot \frac{4\left(m_{a}^{2}+m_{b}^{2}-m_{a+b}^{2}\right)}{\left(\left(m_{a}^{2}+m_{b}^{2}-m_{a+b}^{2}\right)^{2}+4 m_{a}^{2} k^{2}\right)}\right. \\
\left.+\frac{1}{2}\left(\frac{m_{b}}{m}\right)^{2} \cdot \frac{1}{\sqrt{k^{2}+m_{b}^{2}}}\right\} .
\end{gathered}
$$


Unfortunately, it is not possible to evaluate the integral $I_{a}$ analytically; except when $r=1$ in which case the integrand is zero. Actually, this case is of interest since it corresponds to the sine-Gordon theory, and we find

$$
\begin{aligned}
M^{\mathrm{q}} & =M^{\mathrm{cl}}+\Delta M \\
& =\frac{8 m}{\sqrt{2} \beta^{2}}-\frac{\sqrt{2} m}{\pi} .
\end{aligned}
$$

So if we define $M^{\mathrm{cl}}\left(\beta^{2}\right)$ to be the classical mass of the soliton, then to lowest order in the quantum corrections

$$
M^{\mathrm{q}}=M^{\mathrm{cl}}\left(\beta^{2}\right)
$$

with

$$
\beta^{2}=\frac{\beta^{2}}{1-\beta^{2} / 4 \pi},
$$

the well-known result for the sine-Gordon quantum soliton mass. Different quantization approaches show that this result is exact [14].

Returning to the more general theories, we can solve the integral numerically. For a large number of values of $r$ we have found that $I_{a}$ is actually independent of $a$. The first 10 values are presented in the table ${ }^{5}$.

\begin{tabular}{|l|l|}
\hline$r$ & $I$ \\
\hline 1 & 0 \\
2 & -1.814 \\
3 & -1.571 \\
4 & -2.672 \\
5 & -2.721 \\
6 & -3.620 \\
7 & -3.792 \\
8 & -4.593 \\
9 & -5.577 \\
\hline
\end{tabular}

Table: the integral $I(r)$

The important point is not the actual value for $I_{a}$, but the fact that it is independent of $a$. This means that to lowest order the soliton spectrum just receives an overall

\footnotetext{
${ }^{5}$ Numerical integration was performed using Mathematica $^{\mathrm{TM}}$.
} 
renormalization and the masses are still proportional to the masses $m_{a}$. Our final results is

$$
M_{a}^{\mathrm{q}}=m_{a}\left(\frac{2(r+1)}{\beta^{\prime 2}}+I\right)+O\left(\beta^{2}\right),
$$

where $\beta^{\prime}$ is defined in (2.35). $I$ is the constant which only depends on $r$.

\section{Discussion}

We have demonstrated that Toda theories with imaginary coupling constant admit soliton solutions, whose topological charges lie in the set of weights of the fundamental representations of the algebra. Surprisingly the solitons have real classical masses and are stable to perturbation. The gross spectrum of the solitons is the same as that of the fundamental Toda particles. This feature is not spoiled by the quantum theory, to lowest order in $\hbar$, and with the sine-Gordon example in mind, we can speculate that, as a result of the integrability of the theory, the masses in (2.36) are also exact.

In the sine-Gordon theory, the soliton and anti-soliton can form bound states - the socalled breathers. The masses of the breathers can be found using more sophisticated timedependent WKB methods [13]. Remarkably the ground state of the breather spectrum is identified as the fundamental particle of the sine-Gordon theory. It is clearly of interest to repeat the calculation of ref. [13] and establish whether the more general Toda theories admit breather states. One would also like to determine the soliton $S$-matrix. Since the theory is integrable we expect this $S$-matrix to be factorizable, however, the solitons carry topological charge and consequently we do not expect the $S$-matrix to be elastic, i.e. we expect non-trivial scattering in the space of topological charge. It appears that the correct $S$-matrix has the form of a product of the minimal $S$-matrix, which determines the pole structure, and a quantum group $R$-matrix which acts in the space of the topological charge; details of this will be presented elsewhere. When the $S$-matrix has been determined, it should then be possible to determine out whether the solitons can be decoupled from the non-unitary sector of the theory.

We have only considered the $A_{r}$ affine Toda field theories and it would be interesting to know whether the construction of the solitons can be extended to the other simplylaced theories. Initial considerations for the affine theory based on $D_{4}$ indicate that soliton 
solutions do exist, and are also associated to the fundamental representations of the algebra. Furthermore, the classical masses of the solitons are consistent with the conjecture

$$
M_{a}^{\mathrm{cl}}=\frac{2 h}{\beta^{2}} m_{a}
$$

where $h$ is the Coxeter number of the algebra, and the $m_{a}$ 's are the masses of the fundamental particles of the theory; that is $2 m \sqrt{\zeta}$, where $\zeta$ is one of the $r$ eigenvalues of (6).

Soliton-type solutions of the $A_{2}$ affine Toda field theory are also considered in [15]; however, they are not the same as the solutions considered here, since they do not satisfy the classical equations of motion.

I would like to thank Mark de Groot for some helpful comments on the manuscript. This work was supported by NSF PHY90-21984.

\section{References}

[1] A.A. Belavin, A.M. Polyakov and A.B. Zamolodchikov, 'Infinite conformal symmetry in two-dimensional quantum field theory', Nucl. Phys. B241 (1984) 333

[2] V.A. Fateev and A.B. Zamolodchikov, 'Conformal quantum field theory models in two-dimensions having Z(3) symmetry', Nucl. Phys. B280(FS18) (1987) 644

V.A. Fateev and S.L. Lukyakhov, 'The models of two-dimensional conformal quantum field theory with $Z(N)$ symmetry', Int. J. Mod. Phys. A3 (1988) 507

[3] V.A. Fateev and A.B. Zamolodchikov, 'Conformal field theory and purely elastic S-matrices', Int. J. Mod. Phys. A5 (1990) 1025

[4] T. Eguchi and S-K. Yang, 'Deformations of conformal field theories and soliton equations', Phys. Lett. 224B (1989) 373

T.J. Hollowood and P. Mansfield, 'Rational conformal field theories at, and away from, criticality, as Toda theories', Phys. Lett. 226B (1989) 73

[5] A. LeClair, 'Restricted sine-Gordon theory and the minimal conformal series', Phys. Lett. 230B (1989) 103

D. Bernard and A. LeClair, 'The fractional supersymmetric sine-Gordon models', Phys. Lett. 247B (1990) 309

F.A. Smirnov, 'Reductions of the sine-Gordon model as perturbations of conformal 
field theory', Nucl. Phys. B337 (1990) 156; 'The perturbed $c<1$ conformal field theories as reductions of the sine-Gordon model', Int. J. Mod. Phys. A4 (1989) 4213 T. Eguchi and S-K. Yang, 'Sine-Gordon theory at rational values of the coupling constant and minimal conformal models', Phys. Lett. 235B (1990) 282

[6] A.E. Arinstein, V.A. Fateev and A.B. Zamolodchikov, 'Quantum S-matrix of the 1+1-dimensional Toda chain', Phys. Lett. 87B (1979) 389

H.W. Braden, E. Corrigan, P.E. Dorey and R. Sasaki, 'Affine Toda field theory and exact S-matrices', Nucl. Phys. B338 (1990) 689; 'Multiple poles and other features of affine Toda field theory', Nucl. Phys. B356 (1991) 469

[7] P. Dorey, 'Root systems and purely elastic S-matrices', Nucl. Phys. B358 (1991) 654

[8] R. Hirota, 'Direct methods in soliton theory', in 'Soliton' page 157: ed. R.K. Bullough and P.S. Caudrey (1980)

[9] 'Soliton theory: a survey of results', ed. A.P. Fordy, Manchester University Press (1990)

[10] R. Rajaraman, 'Some non-perturbative methods in quantum field theory', Phys. Rep. 21C (1975) 227

[11] R. Hirota, 'Exact solution of the sine-Gordon equation for multiple collisions of solitons', J. Phys. Soc. Japan 33 (1972) 1459

[12] M. Todd, 'Theory of non-linear lattices', Springer series in solid-state sciences 20

[13] R.F. Dashen, B. Hasslacher and A. Neveu, 'Particle spectrum in model field theories from semiclassical functional integral techniques', Phys. Rev. D11 (1975) 3424; 'Nonperturbative methods and extended-hadron models in field theory. II. Two-dimensional models and extended hadrons', Phys. Rev. D10 (1974) 4130

[14] S. Coleman, 'The quantum sine-Gordon equation as the massive Thirring model', Phys. Rev. D11 (1975) 2088

[15] T. Nakatsu, 'Quantum group approach to affine Toda field theory', Nucl. Phys. B356 (1991) 499 